\title{
Knockdown of RHOC by shRNA suppresses invasion and migration of cholangiocellular carcinoma cells via inhibition of MMP2, MMP3, MMP9 and epithelial-mesenchymal transition
}

\author{
HAIXIA YANG ${ }^{1,2^{*}}$, JUN LIANG $^{1,2^{*}}$ JIUPENG ZHOU $^{3}$, JIANQIANG MI $^{4}, \mathrm{KE} \mathrm{MA}^{5}$, \\ YANGWEI FAN ${ }^{5}$, JING NING $^{5}$, CHUYING WANG $^{5}$, XIN WEI $^{5}$ and ENXIAO LI ${ }^{5}$ \\ ${ }^{1}$ Department of Radiotherapy, Tangdu Hospital, Fourth Military Medical University, Xi'an, Shaanxi 710038; \\ ${ }^{2}$ Cancer Institute of the Fourth Military Medical University, Xi'an, Shaanxi 710038; ${ }^{3}$ Department of Medical Oncology, \\ Xi'an Chest Hospital of Shaanxi, Xi'an, Shaanxi 710061; ${ }^{4}$ Department of Pathology, \\ First Affiliated Hospital of Henan Science and Technology University, Luoyang, Henan 471003; \\ ${ }^{5}$ Department of Medical Oncology, First Affiliated Hospital of Xi'an Jiaotong University, \\ Xi'an, Shaanxi 710062, P.R. China
}

Received May 23, 2015; Accepted April 3, 2016

DOI: $10.3892 / \mathrm{mmr} .2016 .5170$

\begin{abstract}
Ras homolog family member C (RHOC) is important during the progression of several types of cancer, including prostate, breast and hepatocellular carcinoma. However, the function of RHOC in cholangiocellular carcinoma (CCC), a highly recurrent and metastatic carcinoma with poor prognosis, remains unclear. The aim of the present study was to investigate the involvement of RHOC in CCC tumor progression. RHOC expression levels were examined in CCC tissues and cells, and adjacent nontumorous bile duct tissues. The effects and molecular mechanisms of RHOC expression on cell migration and invasion were also investigated. The current study demonstrated that RHOC protein was frequently overexpressed in human $\mathrm{CCC}$ specimens and CCC cell lines. Downregulation of RHOC inhibited CCC cell invasion and migration partially via inhibition of matrix metalloproteinase 2, 3 and 9 expression. RHOC also modulated the expression of several epithelial-mesenchymal transition (EMT)-associated proteins, including E-cadherin, vimentin, Slug and Snail, to promote to EMT progression. The present results demonstrated that $\mathrm{RHOC}$ is important for the invasion and migration of CCC through simultaneous regulation of
\end{abstract}

Correspondence to: Professor Enxiao Li, Department of Medical Oncology, First Affiliated Hospital of Xi'an Jiaotong University, 277 Yanta West Road, Xi'an, Shaanxi 710062, P.R. China

E-mail: doclienxiao@126.com

*Contributed equally

Key words: human cholangiocellular carcinoma, RHOC, matrix metalloproteinases, epithelial-mesenchymal transitions, invasion, migration
MMPs and EMT-associated protein, suggesting that RHOC is a potential molecular target for CCC treatment.

\section{Introduction}

Cholangiocellular carcinoma (CCC) is a relatively rare malignant tumor of the bile duct epithelium. In Europe, approximately 10,000 new cases of CCC are diagnosed every year (1). CCC is classified into intrahepatic, perihilar and distal cholangiocarcinoma according to anatomical location. Currently, complete surgical resection is the only means for cure in patients with CCC at the early stage (2). However, most patients are initially diagnosed at the advanced stage, losing the opportunity of radical surgical resection (3). Even following surgery, the 5-year recurrence rate is in the range of $60-90 \%$ (4). The overall 5 year survival rate of patients with $\mathrm{CCC}$ is $<5 \%$ and median survival time is $\sim 12-30$ months (5). Rapid invasion and metastatic capabilities of CCC contribute to the poor prognosis and resistance to the clinical therapeutic strategies (6). Thus, it is necessary to understand the precise mechanisms of this process to elucidate novel therapeutic modalities and improve the prognosis of patients with CCC.

Ras homolog family member C (RHOC) is a member of the ras superfamily of GTP-binding proteins, which act as molecular switches between active GTP-bound and inactive GDP-bound states (7). The family of RHO genes, which are important for cell proliferation and motility, have previously been implicated in tumorigenesis and metastatic progression (8). The RHO subfamily includes RHOA, RHOB and RHOC, which share $85 \%$ amino acid sequence identity (9). Despite this similarity, each protein has differing affinities for various downstream effectors and demonstrate different subcellular localization, suggesting that they have distinct functions in normal cellular activities and during tumor pathogenesis (10). Overexpression of RHOA has previously been reported to promote the invasiveness of tumor cells in several types of malignancy (11). By contrast, RHOB was 
previously reported as a suppressor or negative modifier of cancer progression (12).

A previous study demonstrated RHOC to be correlated with the metastasis of various types of tumor (13). Other studies have demonstrated that RHOC expression is associated with aggressive phenotypes in a human cholangiocarcinoma cell line $(14,15)$. However, the precise molecular mechanisms involved remain unclear. Thus, the current study aimed to investigate the pathological function of RHOC and the potential molecular mechanisms associated with cholangiocarcinoma.

\section{Materials and methods}

Patients and clinicopathological data. Clinical and pathological data were collected from 24 patients that underwent surgical resection of pathologically confirmed CCC between March 10, 2011 and May 15, 2014 at the First Affiliated Hospital of Henan Science and Technology University (Luoyang, China). Demographic data and pathological results were collected for each patient. The study was approved by Ethics Committee of the First Affiliated Hospital of Henan Science and Technology University in March 2011. All patients provided signed informed consent. Additionally, 24 samples of adjacent nontumorous bile duct tissues (NBD) were obtained as controls. All fresh samples were obtained from surgical resection and immediately preserved in liquid nitrogen. Clinicopathological staging was determined by the TNM classification of the 7 th edition American Joint Committee on Cancer (16).

Cells lines and cell culture. RBE and HCCC-9810 human cholangiocarcinoma cell lines were purchased from the Shanghai Institutes for Biological Sciences (Shanghai, China) and Wuhan Boster Biological Technology, Ltd. (Wuhan, China), respectively. QBC939 and SK-ChA-1 human cholangiocarcinoma cell lines were kindly provided by Dr. Chundong Yu (Xiamen University, Xiamen, China). All cell lines were cultured in Dulbecco's modified Eagle's medium or RPMI 1640 medium supplemented with $10 \%$ fetal bovine serum (FBS), streptomycin $(100 \mu \mathrm{g} / \mathrm{ml})$ and penicillin (100 units/ml) purchased from Hyclone; Thermo Fisher Scientific, Inc. (Logan, UT, USA) at $37^{\circ} \mathrm{C}$ in $5 \% \mathrm{CO}_{2}$ atmosphere.

Lentivirus vector and cell transfection. Lentiviral-mediated RHOC short hairpin RNA (shRNA) and negative control shRNA were packaged and produced by Shanghai GenePharma Co., Ltd. (Shanghai, China). The RHOC shRNA target sequence (NM_175744, NCBI GenBank accession number) was cloned into the pGLV-3/H1/GFP + puro lentiviral vector (Shanghai GenePharma Co., Ltd.), which specifically expresses RHOC shRNA. The RHOC shRNA target sequence was as follows: 5'-GATCCCGCTATATTGCGG ACATTGAGTTCAAGAGACTCAATGTCCGCAATATAG TTTTTTGGAAA-3', as described by Wu et al (17). The shRNA nontarget sequence, 5'-TTCTCCGAACGTGTC ACGT-3' was also cloned into a pGLV-3/H1/GFP + puro lentiviral vector as a negative control. The recombinant lentivirus RHOC shRNA (Lv-shRHOC) and control shRNA
(Lv-shCTRL) were packaged in 293T cells (Shanghai GenePharma Co., Ltd.) using a lentivector expression system. DNA sequencing results revealed that the shRNA interference sequence targeting the RHOC gene was successfully inserted into the recombinant lentivirus. For cell infection, $30-50 \%$ confluent RBE and HCCC-9810 negative control shRNA and RHOC shRNA cell lines were incubated with lentivirus for 72-96 h. Untransfected controls were used in preliminary shRNA experiments, and demonstrated no significant difference compared with the negative control shRNA group. Thus, negative control shRNA was used to represent normal controls in the subsequent experiments The expression of green fluorescent protein (GFP) was examined under an Eclipse Ti fluorescence microscope (Nikon Corporation, Tokyo, Japan), and the intensity of green fluorescence indicated the transduction efficiency. The CCC cells were transfected with high titres of Lv-shRHOC and LV-shCTRL particles $\left(2 \times 10^{8} \mathrm{TU} / \mathrm{ml}\right.$; MOI of 30 transfection concentration) according to the instructions of the lentivirus manufacturer. Stable knockdown of RHOC and negative control shRNA transfectants were obtained by continuous treatment with $2 \mu \mathrm{g} / \mathrm{ml}$ puromycin (Shanghai GenePharma Co., Ltd.) in transfected RBE and HCCC-9810 cell lines.

Western blotting assay. Proteins were extracted from the cells using radioimmunoprecipitation assay lysis buffer (Nanjing KeyGen Biotech Co., Ltd., Nanjing, China). Lysates were centrifuged at $4^{\circ} \mathrm{C}$ at $4,000 \times \mathrm{g}$ for $20 \mathrm{~min}$, and the supernatants were collected. Protein concentration was quantified using a Bradford assay (Beyotime Institute of Biotechnology, Haimen, China). Lysates $(50 \mu \mathrm{g})$ were separated by $10 \%$ sodium dodecyl sulfate-polyacrylamide gel (Beyotime Institute of Biotechnology) electrophoresis and transferred onto nitrocellulose membrane (EMD Millipore, Billerica, MA, USA). Membranes were blocked by $5 \%$ non-fat milk in Tris-buffered saline Tween 20 (TBST) then incubated with primary antibodies at $4-8^{\circ} \mathrm{C}$ overnight. The primary antibodies used were monoclonal mouse anti-RHOC (1:300 dilution; cat. no. AT3636a; Abgent, Inc., San Diego, CA, USA), monoclonal mouse anti-MMP2, MMP3 and MMP9 (all 1:1,000 dilution; cat. nos. ab-86607, ab-17790 and ab-58803, respectively; Abcam, Cambridge, MA, USA), monoclonal mouse anti-MMP14 (1:2,000 dilution; cat. no. ab-78738; Abcam), polyclonal rabbit anti-E-cadherin, Vimentin, Snail and Slug (all 1:500 dilution; cat. nos. WL-01482, WL-01960, WL-01863 and WL-01508, respectively; Wanleibio Co., Ltd., Shenyang, China) and monoclonal mouse anti- $\beta$-actin (1:5,000 dilution; cat. no. sc-47778; Santa Cruz Biotechnology, Inc., Dallas, TX, USA). Membranes were washed with TBST 3 times for 10 min then incubated with horseradish peroxidase-conjugated goat anti-rabbit (cat. no. sc-2004) and goat anti-mouse (cat. no. sc-2005) secondary antibodies (1:3,000 dilution; Santa Cruz Biotechnology, Inc.) for $1 \mathrm{~h}$ at room temperature. Protein levels were determined by normalizing to $\beta$-actin. The proteins were visualized by enhanced chemiluminescence (Advansta, Inc., Menlo Park, CA, USA) and detected using Bio-Rad ChemiDoc MP imaging system (ImageLab 4.1 software; Bio-Rad Laboratories, Inc., Hercules, CA, USA). The experiments were performed in triplicate. 
Table I. Clinicopathological information of cholangiocellular carcinoma cases studied and RHOC expression in cholangiocellular carcinoma tissue.

\begin{tabular}{|c|c|c|c|c|c|c|}
\hline Case no. & Gender & Age years & Type & Stage $^{b}$ & Differentiation & $\begin{array}{l}\text { RHOC expression } \\
\left.\text { (Fold change }{ }^{\mathrm{a}}\right)\end{array}$ \\
\hline 1 & Male & 65 & Intrahepatic & T2aNxMx & NA & 1.81 \\
\hline 2 & Female & 45 & Extrahepatic & T3N1M0 & NA & 1.93 \\
\hline 3 & Male & 47 & Intrahepatic & T1N0M0 & NA & 1.02 \\
\hline 4 & Male & 34 & Extrahepatic & T3N2M0 & NA & 6.21 \\
\hline 5 & Male & 29 & Intrahepatic & T4N2M0 & NA & 9.45 \\
\hline 6 & Female & 67 & Extrahepatic & T3N0M0 & Well & 0.67 \\
\hline 7 & Male & 71 & Extrahepatic & NA & Poor & 3.15 \\
\hline 8 & Female & 58 & Intrahepatic & T2aNxMx & Poor & 5.17 \\
\hline 9 & Male & 49 & Extrahepatic & T2bNOM0 & Well & 1.10 \\
\hline 10 & Male & 44 & Intrahepatic & T3N1M0 & Moderately & 4.23 \\
\hline 11 & Male & 73 & Extrahepatic & T1N0M0 & Well & 0.77 \\
\hline 12 & Female & 54 & Extrahepatic & $\mathrm{T} 2 \mathrm{aN} 2 \mathrm{M} 0$ & Moderately & 4.89 \\
\hline 13 & Male & 65 & Extrahepatic & T1N1M0 & Moderately & 3.78 \\
\hline 14 & Male & 41 & Intrahepatic & TxN1M0 & Poor & 2.56 \\
\hline 15 & Male & 38 & Intrahepatic & T1N1M0 & Moderately & 2.66 \\
\hline 16 & Female & 44 & Extrahepatic & NA & Poor & 5.66 \\
\hline 17 & Male & 57 & Extrahepatic & T2bN0M0 & Well & 0.57 \\
\hline 18 & Female & 55 & Extrahepatic & NA & Moderately & 3.40 \\
\hline 19 & Male & 63 & Extrahepatic & $\mathrm{T} 2 \mathrm{bN} 2 \mathrm{M} 0$ & Moderately & 7.34 \\
\hline 20 & Female & 68 & Intrahepatic & T3N1M1 & Moderately & 2.91 \\
\hline 21 & Male & 36 & Extrahepatic & T2bN1M0 & Moderately & 3.35 \\
\hline 22 & Female & 47 & Intrahepatic & T4N2M0 & Poor & 6.23 \\
\hline 23 & Male & 36 & Extrahepatic & $\mathrm{T} 2 \mathrm{aN} 1 \mathrm{M} 0$ & Well & 1.88 \\
\hline 24 & Female & 42 & Extrahepatic & T1N0M0 & Moderately & 1.67 \\
\hline
\end{tabular}

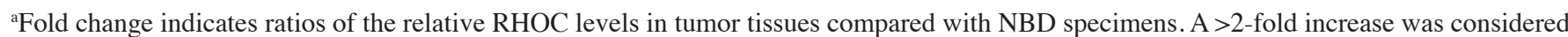
as RHOC upregulation. The result of paired t-test demonstrated that RHOC protein was overexpressed in tumor tissues compared with NBD $(\mathrm{t}=7.199, \mathrm{P}<0.001)$. ${ }^{\mathrm{b}}$ Stages were classified according to the American Joint Committee on Cancer TNM system. RHOC, ras homolog family member C; NBD, nontumorous bile duct; NA, not available.

A
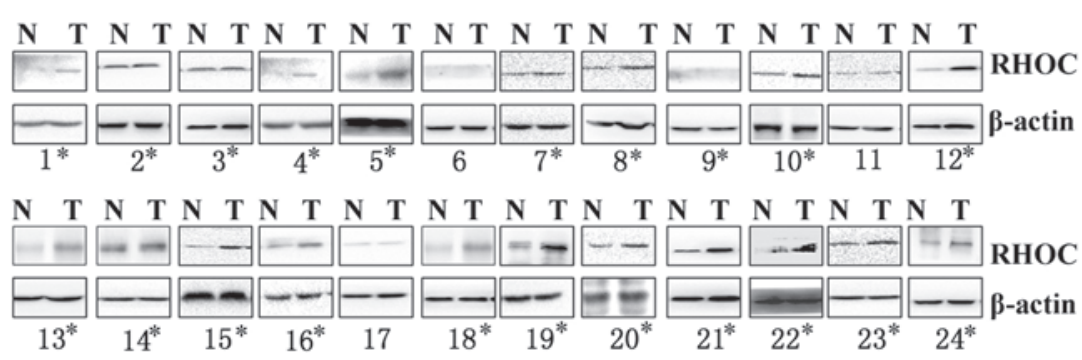

T N T

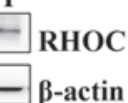

B

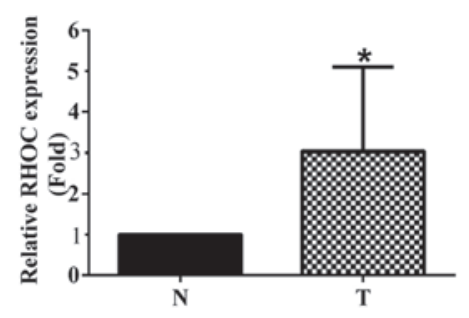

Figure 1. Overexpressed RHOC in human CCC tissues. (A) Expression of RHOC protein in human CCC specimens and adjacent $\mathrm{N}$ tissues. ${ }^{*} \mathrm{P}<0.05$ indicates significant overexpression of RHOC in CCC specimens. Western blot analysis was repeated in triplicate. (B) The protein levels of RHOC were significantly higher in CCC specimens compared with $\mathrm{N}$ specimens. Values are presented as the mean \pm standard deviation. $\mathrm{P}=0.000$ vs. $\mathrm{N}$. RHOC, ras homolog family member C; CCC, cholangiocellular carcinoma; N, nontumorous bile duct; $\mathrm{T}$, tumor.

In vitro invasion assay. For the invasion assay, $3 \times 10^{4}$ cells were added to the cell culture inserts with microporous membrane and Matrigel coating (BD Biosciences, Franklin Lakes, NJ, USA). Medium containing $10 \%$ FBS was added to the bottom chamber. The cells were then incubated for $48 \mathrm{~h}$ at $37^{\circ} \mathrm{C}$ and the upper chamber was removed. The cells on the bottom surface of the upper chambers fixed in $95 \%$ ethanol (Sangon Biotech Co., Ltd., Shanghai, China) for $15 \mathrm{~min}$ and stained with $0.1 \mathrm{mg} / \mathrm{ml}$ crystal violet solution and the number of cells was counted under a Eclipse Ti microscope (magnification, $\mathrm{x} 200$ ). Individual experiments had triplicate inserts and five randomly selected fields were counted per insert. 


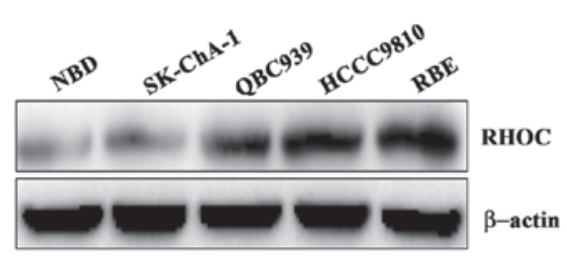

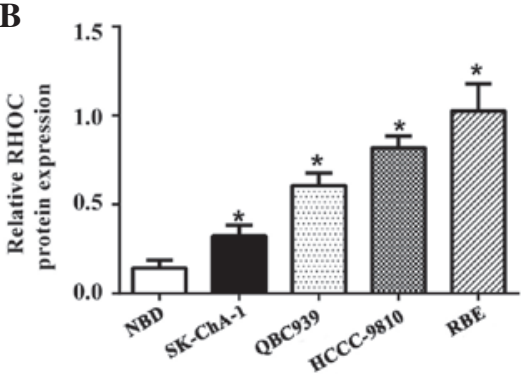

Figure 2. RHOC is overexpressed in human CCC cell lines. (A) Expression of RHOC protein in human CCC cell lines (SK-ChA-1, QBC939, HCCC9810 and RBE) and adjacent NBD samples. (B) Compared with NBD epithelium, RHOC expression was significantly increased in the 4 CCC cell lines. "P<0.01 vs. NBD. Values are presented as the mean \pm standard deviation. RHOC, ras homolog family member C; CCC, cholangiocellular carcinoma; NBD, nontumorous bile duct.

A
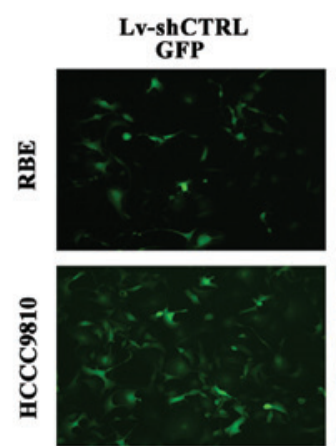

C
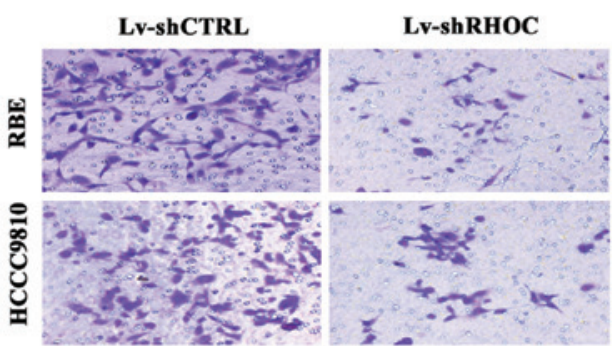

D

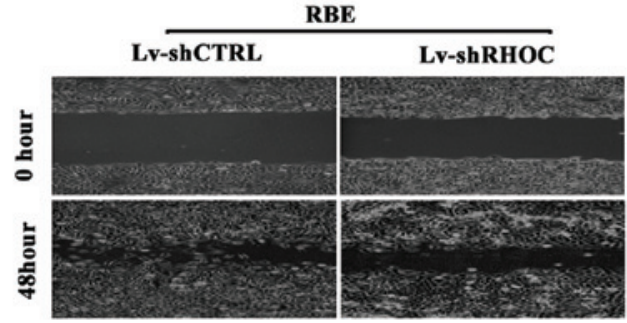

$\mathbf{E}$
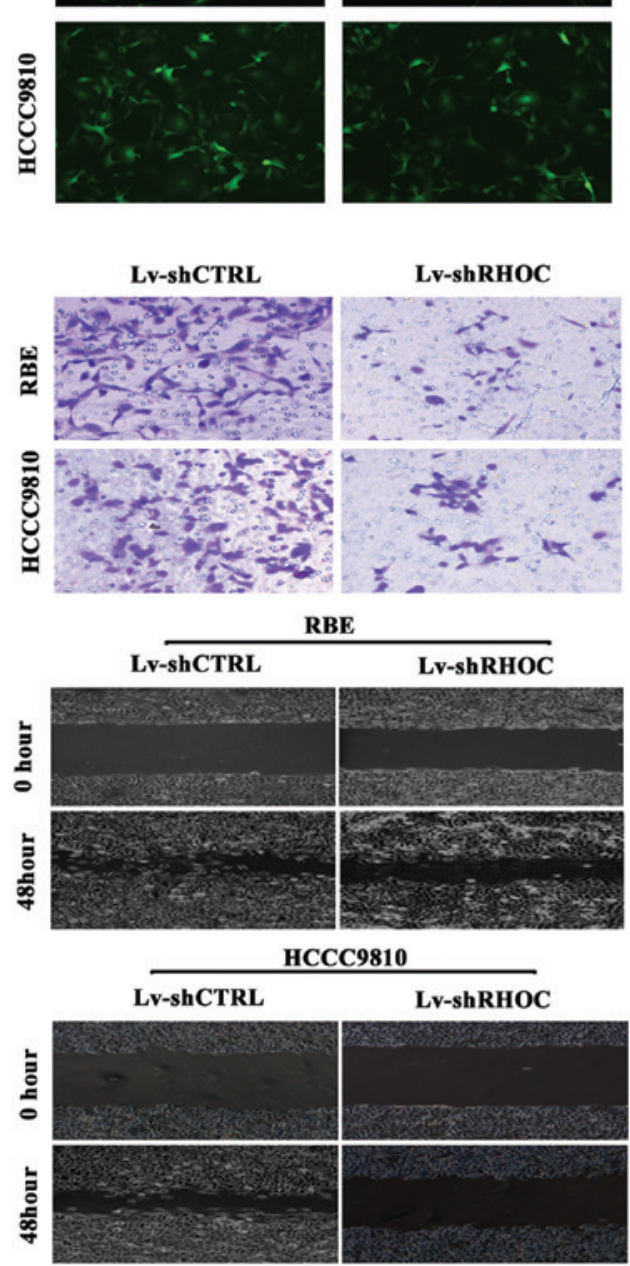

B
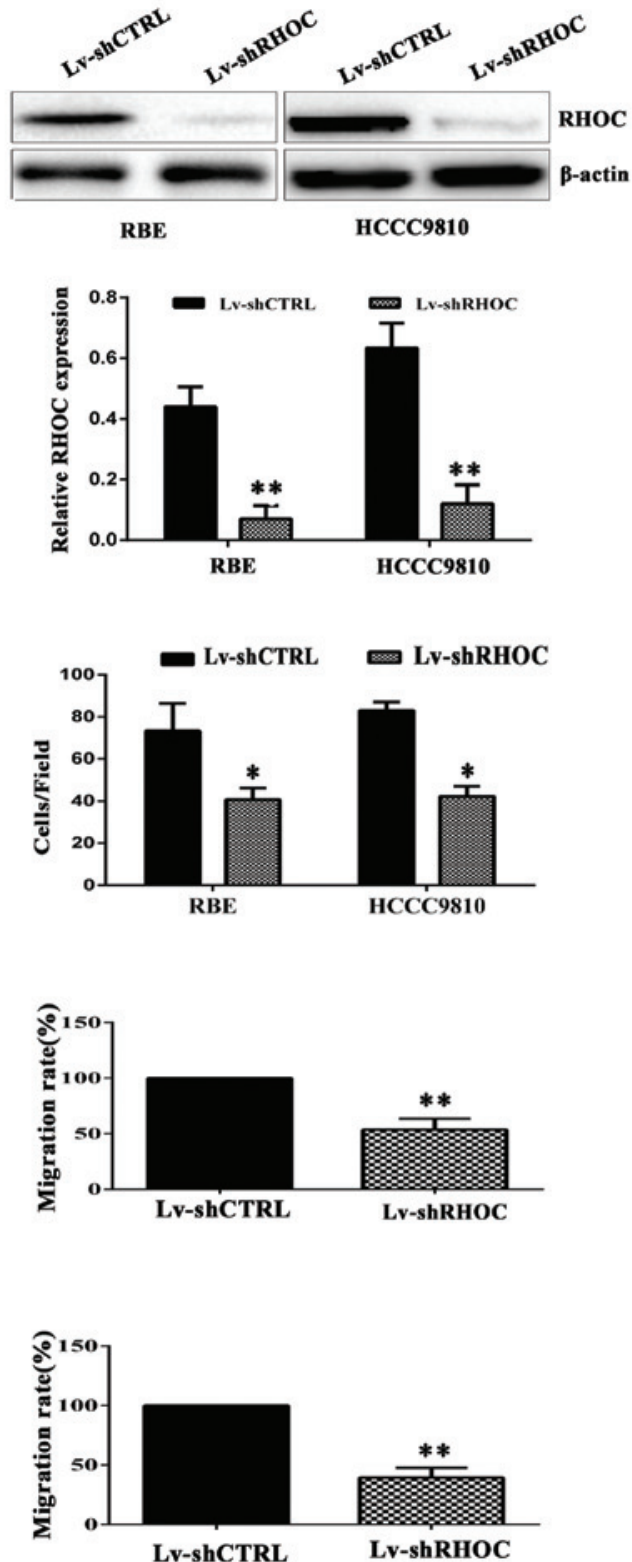

Figure 3. Effect of RHOC knockdown on RBE and HCCC-9810 cell invasion and migration. (A) Lentiviral transduction efficiency. Fluorescence microscopy (magnification, $\mathrm{x} 100$ ) demonstrated $>95 \%$ RBE and HCCC-9810 cells were effectively transfected with Lv-shRHOC and Lv-shCTRL vector 4 days after transfection at a multiplicity of infection of 30. (B) Significantly decreased RHOC protein expression following lentiviral shRNA stable transfection. (C) Cell invasion capacity of transfected RBE and HCCC-9810 cells by a Transwell assay and quantification of the number of invaded RBE and HCCC-9810 cells in each group. Scratch assays showed that RHOC shRNA knockdown (D) RBE and (E) HCCC9810 cells had reduced motility compared with control cells. Representative images are presented and quantification of mean migration rates of RBE and HCCC-9810 cells in each group. The data are presented as the mean \pm standard deviation of three independent experiments. ${ }^{*} \mathrm{P}<0.05,{ }^{* *} \mathrm{P}<0.01 \mathrm{vs}$. Lv-shCTRL. RHOC, Ras homolog family member C; Lv, lentivirus; Lv-shRHOC, RHOC small hairpin RNA interference group; Lv-shCTRL, nontarget shRNA interference group; GFP, green fluorescent protein. 
Scratch assay. For wound-healing assays, RBE and HCCC 9810 cells were plated at $2 \times 10^{6}$ cells/dish density in $60 \mathrm{~mm}$-diameter dishes. Wounds were created in the confluent cells using a $200 \mu \mathrm{l}$ pipette tip after cells had reached confluency. The cells were then rinsed with medium to remove free-floating cells and debris. Medium with $1 \%$ FBS was added and the culture plates were incubated at $37^{\circ} \mathrm{C}$ for $48 \mathrm{~h}$. Different stages of wound healing were observed along the scrape line and representative scrape lines were imaged with an Eclipse Ti microscope. Wound closure was measured using AxioVision software version 4.7 (Zeiss GmbH Jena, Germany). Quantification was performed by measuring the uncovered areas compared with the controls. Each experiment was repeated in triplicate.

Statistical analysis. All data were analyzed for statistical significance using SPSS software, version 18.0 (SPSS, Inc., Chicago, IL, USA). The data are expressed as the mean \pm standard deviation. The protein expression levels were measured using densitometry with ImageLab 4.1 software. Two-tailed Student's t-test was used for comparisons of two independent groups, and analysis of variance followed by Dunnett's test was used for multiple comparisons. $\mathrm{P}<0.05$ was considered to indicate a statistically significant difference.

\section{Results}

RHOC is highly expressed in human CCC tissues and cell lines. To evaluate the expression of RHOC in CCC, western blotting was performed to assess the levels of RHOC protein in 24 tumor and 24 adjacent NBD tissues. As presented in Fig. 1 and Table I, the levels of RHOC protein were significantly upregulated in $21 \mathrm{CCC}$ specimens, including 12 extrahepatic CCC and 9 intrahepatic CCC samples, compared with NBD specimens $(\mathrm{P}=0.000)$. Additionally, RHOC expression was significantly increased in CCC cell lines, including HCCC9810, QBC939, RBE and SK-ChA-1, compared with NBD epithelium (Fig. 2; $\mathrm{P}=0.006$ ). Thus, the overexpression of RHOC in CCC specimens and CCC cell lines suggests that RHOC may be important for the tumorigenesis of CCC.

RHOC protein expression is inhibited by lentiviral-mediated shRNA interference in RBE and HCCC-9810 cell lines. Analysis of western blotting results demonstrated that the relative protein expression levels of RHOC were 1-3-fold higher in RBE and HCCC-9810 cells compared with QBC939 and SK-ChA-1 cell lines (Fig. 2). Thus, the RBE and HCCC-9810 cell lines were selected for use in knockdown experiments. The cells were transfected with Lv-shRHOC and Lv-shCTRL lentiviral vectors. The transfection efficiency of $>90 \%$ was determined by detecting the expression of GFP $96 \mathrm{~h}$ after infection (Fig. 3A). Western blotting indicated RHOC was significantly downregulated in RBE and HCCC-9810 cells transfected with Lv-shRHOC vector compared with Lv-shCTRL. In comparison with Lv-shCTRL, the protein levels of RHOC were decreased by $83.73 \pm 9.09 \%$ and $81.2 \pm 8.53 \%$ in $\mathrm{Lv}$-shRHOC-transfected RBE and HCCC-9810 cells, respectively ( $\mathrm{P}<0.003$, Fig. 3B).

RHOC silencing impairs CCC cell invasion and migration in vitro. The results of the Matrigel invasion assay indicated
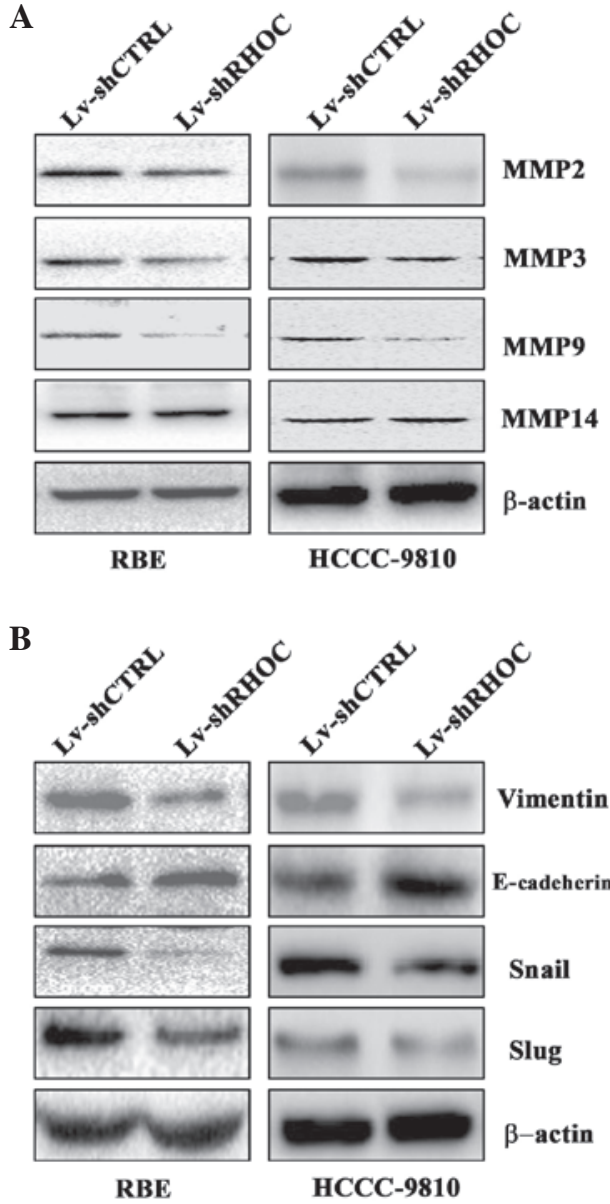

Figure 4. Expression levels of MMPs and epithelial mesenchymal transition-associated genes in RBE and HCCC-9810 cells following knockdown of RHOC. (A) The relative protein levels of MMP2 and 9 were decreased, and the expression levels of MMP3 and 9 were unchanged in LV-shRHOC transfected RBE and HCCC-9810 cells compared with control. (B) Protein expression levels of vimentin, Snail and Slug were reduced, and E-cadherin increased in RBE and HCCC-9810 cells compared with control. MMP, matrix metalloproteinase; RHOC, ras homolog family member C; Lv, lentivirus; Lv-shRHOC, RHOC small hairpin RNA interference group; Lv-shCTRL, nontarget shRNA interference group.

that inhibition of RHOC reduced the invasive ability of $\mathrm{RBE}$ and HCCC-9810 cells compared with Lv-shCTRL-transfected cells $(\mathrm{P}<0.016$; Fig. 3C). The effects of RHOC on the migration of CCC cell lines were assessed by scratch assay. The results demonstrated that compared with Lv-shCTRL-transfected cells, RHOC knockdown significantly reduced cell migration in RBE and HCCC-9810 cells ( $\mathrm{P}<0.003$; Fig. 3D and $\mathrm{E})$. Collectively, these findings provided evidence that elevated RHOC expression levels were involved in promoting the migratory and invasive phenotype of CCC cells.

Effects of RHOC knockdown on the expression of tumor invasion-associated molecules. As the invasive ability of tumor cells is often correlated with the production of secretory proteases, the effect of RHOC knockdown on the expression of tumor invasion-associated molecules was determined. As demonstrated in Fig. 4A, western blotting analysis indicated that downregulation of $\mathrm{RHOC}$ reduced the protein expression level of MMP2, 3 and 9, whereas, no change was observed in the expression level of MMP14 (Fig. 4A). 
Effects of RHOC knockdown on the expression of epithelial mesenchymal transition (EMT)-associated genes in CCC cell lines. To further investigate the mechanism by which RHOC regulates cell invasion and migration, the protein expression levels of EMT-associated genes in CCC cells was analyzed. It was demonstrated that RHOC knockdown decreased the expression of the mesenchymal marker, vimentin, and the EMT central regulators, Snail and Slug, compared with Lv-shCTRL-transfected cells. In contrast to Lv-shCTRL-transfected cells, the expression of epithelial marker E-cadherin was upregulated in CCC cell lines following RHOC knockdown (Fig. 4B). Thus, the results of the current study demonstrated that knockdown of RHOC altered the expression levels of MMP2, 3 and 9, and EMT-associated genes, therefore, suppressing the invasive and migratory phenotype of CCC cells.

\section{Discussion}

CCC is a highly metastatic disease characterized by invasive growth along the lymphangion or perineurium, or direct invasion into the liver (18). Thus, intensive studies for potential candidate molecules involved in the metastatic process are urgently required to provide effective treatments for patients with advanced CCC.

RHOC has been shown to promote cancer metastasis in a variety of tumor types, including breast cancer (19), non-small cell lung carcinoma (20), colon carcinoma (21), malignant melanoma (22), hepatocellular carcinoma (23), head and neck cancer (24) and prostate cancer (25). However, the function of RHOC in human CCC remains unclear. The current study demonstrated that the expression levels of RHOC were significantly increased in CCC tissues and cell lines compared with normal biliary epithelium, suggesting that RHOC may be involved in the progression of CCC. Additionally, the present study specifically and efficiently inhibited RHOC gene expression in CCC cell lines by lentiviral-mediated shRNA interference, and the results demonstrated that the invasion and migration capacities of transfected RBE and HCCC9810 cell lines were significantly inhibited by RHOC knockdown in vitro. These results collectively suggested that RHOC may be important in CCC progression.

To date, the molecular mechanisms by which RHOC promote tumor development and metastasis are not fully understood (26). Previous studies have demonstrated that RHOC promote cancer progression by regulating the expression of MMP genes $(14,23,27)$. It is well established that MMPs induce cancer cell invasion and metastatic spread by degrading the extracellular matrix and other barriers (28). In hepatocellular carcinoma, Liao et al (29) reported that hepatocellular carcinoma cell invasion and migration are modulated by the genes RHOC, MMP-2 and MMP-9 (29). Wang et al (14) also demonstrated that RHOC promotes the malignant progression of CCC cells via regulation of MMP expression levels (14). The present study demonstrated that RHOC promotes CCC cell invasion partially via inducing the expression of MMP2, 3 and 9, but not that of MMP14, which is consistent with previous observations in other types of tumor (27,30,31). Further studies are required to determine the precise molecular mechanisms of the invasion process in $\mathrm{CCC}$.
Emerging evidence has established that EMT is an important event during carcinoma progression (32). EMT promotes carcinoma progression by increasing migratory and invasive properties, and cancer stem cell-like phenotype of cells, which may be prerequisites for cancer cell metastasis (32). Thus, understanding the regulatory mechanisms of EMT may provide greater insight into the signaling programs that control CCC metastasis. EMT is characterized by decreased expression of epithelial proteins, such as E-cadherin, and increased expression of mesenchymal proteins, such as vimentin (32). Additionally, Snail and Slug are crucial for the transcriptional regulation of EMT $(33,34)$. Reportedly, either upregulation or increased activity of RHOC promotes the invasive potential of cancer cells, which is closely associated with EMT (35). Bellovin et al (21) reported that RHOC expression and activation are induced by EMT and RHOC promotes post-EMT cell migration. In ovarian carcinoma, a study by Gou et al (36) indicated that ectopic RHOC expression enhanced migration, invasion and altered the expression of EMT markers. Similarly, the current study in CCC demonstrated that RHOC knockdown markedly altered the expression of EMT-associated genes. These results consistently suggested that RHOC has an important function during the malignant progression of $\mathrm{CCC}$ by regulating MMPs and EMT.

In summary, the results of the present study indicate that the expression of RHOC is significantly increased in CCC cell lines and clinical samples. Furthermore, knockdown of RHOC expression significantly inhibited CCC cell migration and invasion in vitro, and regulated the expression of MMPs and EMT-associated genes. Thus, strategies interfering with RHOC expression may provide a novel and promising alternative approach for the treatment of aggressive CCC.

\section{Acknowledgements}

The present study was supported by the National Natural Science Foundation of China (no. 81172026).

\section{References}

1. Razumilava N and Gores GJ: Cholangiocarcinoma. Lancet 383: 2168-2179, 2014.

2. Vogel A, Wege H, Caca K, Nashan B and Neumann U: The diagnosis and treatment of cholangiocarcinoma. Dtsch Arztebl Int 111: 748-754, 2014.

3. Ghouri YA, Mian I and Blechacz B: Cancer review: Cholangiocarcinoma. J Carcinog 14: 1, 2015.

4. Groot Koerkamp B and Fong Y: Outcomes in biliary malignancy. J Surg Oncol 110: 585-591, 2014.

5. Huang Y, Li X and Zhao Y: Progression of targeted therapy in advanced cholangiocarcinoma. Chin J Cancer Res 27: 122-127, 2015.

6. Sirica AE: Cholangiocarcinoma: Molecular targeting strategies for chemoprevention and therapy. Hepatology 41: 5-15, 2005.

7. Turner SJ, Zhuang S, Zhang T, Boss GR and Pilz RB: Effects of lovastatin on Rho isoform expression, activity and association with guanine nucleotide dissociation inhibitors. Biochem Pharmacol 75: 405-413, 2008.

8. Amin E, Dubey BN, Zhang SC, Gremer L, Dvorsky R, Moll JM, Taha MS, Nagel-Steger L, Piekorz RP, Somlyo AV and Ahmadian MR: Rho-kinase: Regulation, (dys) function and inhibition. Biol Chem 394: 1399-1410, 2013.

9. Schaefer A, Reinhard NR and Hordijk PL: Toward understanding RhoGTPase specificity: Structure, function and local activation. Small GTPases 5: 6, 2014. 
10. Donnelly SK, Bravo-Cordero JJ and Hodgson L: Rho GTPase isoforms in cell motility: Don't fret, we have FRET. Cell Adh Migr 8: 526-534, 2014.

11. O'Connor K and Chen M: Dynamic functions of RhoA in tumor cell migration and invasion. Small GTPases 4: 141-147, 2013.

12. Vega FM, Thomas M, Reymond $\mathrm{N}$ and Ridley AJ: The Rho GTPase RhoB regulates cadherin expression and epithelial cell-cell interaction. Cell Commun Signal 13: 6, 2015.

13. Bravo-Cordero JJ, Hodgson L and Condeelis JS: Spatial regulation of tumor cell protrusions by RhoC. Cell Adh Migr 8: 263-267, 2014

14. Wang Q, Tang H, Yin S and Dong C: Downregulation of microRNA-138 enhances the proliferation, migration and invasion of cholangiocarcinoma cells through the upregulation of RhoC/p-ERK/MMP-2/MMP-9. Oncol Rep 29: 2046-2052, 2013

15. Shi Z, Chen ML, He QL and Zeng JH: Antisense RhoC gene suppresses proliferation and invasion capacity of human QBC939 cholangiocarcinoma cells. Hepatobiliary Pancreat Dis Int 6: 516-520, 2007.

16. Edge SB and Compton CC: The American joint committee on cancer: The 7th edition of the AJCC cancer staging manual and the future of TNM. Ann Surg Oncol 17: 1471-1474, 2010.

17. Wu M, Wu ZF, Rosenthal DT, Rhee EM and Merajver SD: Characterization of the roles of RHOC and RHOA GTPases in invasion, motility and matrix adhesion in inflammatory and aggressive breast cancers. Cancer 116: 2768-2782, 2010.

18. Skipworth JR, Keane MG and Pereira SP: Update on the management of cholangiocarcinoma. Dig Dis 32: 570-578, 2014.

19. Rosenthal DT, Zhang J, Bao L, Zhu L, Wu Z, Toy K, Kleer CG and Merajver SD: RhoC impacts the metastatic potential and abundance of breast cancer stem cells. PLoS One 7: e40979, 2012.

20. Shikada Y, Yoshino I, Okamoto T, Fukuyama S, Kameyama T and Maehara Y: Higher expression of RhoC is related to invasiveness in non-small cell lung carcinoma. Clin Cancer Res 9: 5282-5286, 2003.

21. Bellovin DI, Simpson KJ, Danilov T, Maynard E, Rimm DL, Oettgen P and Mercurio AM: Reciprocal regulation of RhoA and RhoC characterizes the EMT and identifies RhoC as a prognostic marker of colon carcinoma. Oncogene 25: 6959-6967, 2006.

22. Goundiam O, Nagel MD and Vayssade M: Akt and RhoA inhibition promotes anoikis of aggregated B16F10 melanoma cells Cell Biol Int 36: 311-319, 2012.

23. Xie S, Zhu M, Lv G, Geng Y, Chen G, Ma J and Wang G: Overexpression of Ras homologous $\mathrm{C}$ (RhoC) induces malignant transformation of hepatocytes in vitro and in nude mouse xenografts. PLoS One 8: e54493, 2013.

24. Islam M, Sharma S, Kumar B and Teknos TN: Atorvastatin inhibits RhoC function and limits head and neck cancer metastasis. Oral Oncol 49: 778-786, 2013.
25. Iiizumi M, Bandyopadhyay S, Pai SK, Watabe M, Hirota S, Hosobe S, Tsukada T, Miura K, Saito K, Furuta E, et al: RhoC promotes metastasis via activation of the Pyk2 pathway in prostate cancer. Cancer Res 68: 7613-7620, 2008.

26. Julian L and Olson MF: Rho-associated coiled-coil containing kinases (ROCK): Structure, regulation and functions. Small GTPases 5: e29846, 2014.

27. Xue F, Takahara T, Yata Y, Xia Q, Nonome K, Shinno E, Kanayama M, Takahara S and Sugiyama T: Blockade of Rho/Rho-associated coiled coil-forming kinase signaling can prevent progression of hepatocellular carcinoma in matrix metalloproteinase-dependent manner. Hepatol Res 38: 810-817, 2008.

28. Cockett MI, Murphy G, Birch ML, O'Connell JP, Crabbe T, Millican AT, Hart IR and Docherty AJ: Matrix metalloproteinases and metastatic cancer. Biochem Soc Symp 63: 295-313, 1998.

29. Liao CG, Kong LM, Zhou P, Yang XL, Huang JG, Zhang HL and $\mathrm{Lu} \mathrm{N}$ : miR-10b is overexpressed in hepatocellular carcinoma and promotes cell proliferation, migration and invasion through RhoC, uPAR and MMPs. J Transl Med 12: 234, 2014.

30. Faried A, Faried LS, Kimura H, Nakajima M, Sohda M, Miyazaki T, Kato H, Usman N and Kuwano $\mathrm{H}$ : RhoA and RhoC proteins promote both cell proliferation and cell invasion of human oesophageal squamous cell carcinoma cell lines in vitro and in vivo. Eur J Cancer 42: 1455-1465, 2006.

31. Ikoma T, Takahashi T, Nagano S, Li YM, Ohno Y, Ando K, Fujiwara T, Fujiwara $\mathrm{H}$ and Kosai $\mathrm{K}$ : A definitive role of RhoC in metastasis of orthotopic lung cancer in mice. Clin Cancer Res 10: 1192-1200, 2004.

32. Kothari AN, Mi Z, Zapf M and Kuo PC: Novel clinical therapeutics targeting the epithelial to mesenchymal transition. Clin Transl Med 3: 35, 2014.

33. Kaufhold S and Bonavida B: Central role of Snaill in the regulation of EMT and resistance in cancer: A target for therapeutic intervention. J Exp Clin Cancer Res 33: 62, 2014.

34. Alves CC, Carneiro F, Hoefler $\mathrm{H}$ and Becker KF: Role of the epithelial-mesenchymal transition regulator slug in primary human cancers. Front Biosci (Landmark Ed) 14: 3035-3050, 2009.

35. Sequeira L, Dubyk CW, Riesenberger TA, Cooper CR and van Golen KL: Rho GTPases in PC-3 prostate cancer cell morphology, invasion and tumor cell diapedesis. Clin Exp Metastasis 25: 569-579, 2008

36. Gou WF, Zhao Y, Lu H, Yang XF, Xiu YL, Zhao S, Liu JM, Zhu ZT, Sun HZ, Liu YP, et al: The role of RhoC in epithelial-to-mesenchymal transition of ovarian carcinoma cells. BMC Cancer 14: 477, 2014. 